\title{
Expiry Prediction and Reducing Food Wastage using loT and ML
}

\author{
Original Scientific Paper
}

\author{
Kartik Nair \\ Dwarkadas J. Sanghvi College of Engineering, \\ Student of Electronics and Telecommunication, \\ Department of Electronics and Telecommunication \\ Mumbai, India \\ kartiknair1999@gmail.com

\section{Bhavya Sekhani} \\ Dwarkadas J. Sanghvi College of Engineering, \\ Student of Electronics and Telecommunication, \\ Department of Electronics and Telecommunication \\ Mumbai, India \\ bhavyasekhani999@gmail.com
}

\author{
Krina Shah \\ Dwarkadas J. Sanghvi College of Engineering, \\ Student of Electronics and Telecommunication, \\ Department of Electronics and Telecommunication \\ Mumbai, India \\ krinas393@gmail.com

\section{Dr. Sunil Karamchandani \\ Dwarkadas J. Sanghvi College of Engineering, Faculty of Electronics and Telecommunication, Department of Electronics and Telecommunication Mumbai, India sunil.karamchandani@djsce.ac.in}

\begin{abstract}
This paper details development of a low-cost, small-size, and portable electronic nose (E-nose) for the prediction of the expiry date of food products. The Sensor array is composed of commercially available metal oxide semiconductors sensors like MQ2 sensor, temperature sensor, and humidity sensor, which were interfaced with the help of ESP8266 and Arduino Uno for data acquisition, storage, and analysis of the dataset consisting of the odor from the fruit at different ripening stages. The developed system is used to analyze gas sensor values from various fruits like bananas and tomatoes. Responding signals of the e-nose were extracted and analyzed. Based on the obtained data we applied a few machine learning algorithms to predict if a banana is stale or not. Logistic regression, Decision Tree Classifier, Support Vector Classifier (SVC) \& K-Nearest Neighbours (KNN) classifiers were the binary classification algorithms used to determine whether the fruit became stale or not. We achieved an accuracy of $97.05 \%$. These results prove that e-nose has the potential of assessing fruits and vegetable freshness and predict their expiry date, thus reducing food wastage.
\end{abstract}

Keywords - electronic nose (E-nose), MQ2 sensor, food expiry prediction, food wastage, machine learning

\section{INTRODUCTION}

Today, many food products come with labels - "Best By" or "Use By" stamped on the packaging. But what do they mean? $37 \%$ of users reported that they discard food each time it's past the expiry date - however the date only denotes peak quality determined by the manufacturer. The manufacturer tests the food in a controlled environment. However, during transportation from Farm-to-Fridge, it undergoes mishandling and temperature fluctuations. Thus, the expiry date mentioned on the packaging is not accurate anymore and this ambiguity leads to food wastage in large quantities around the world, daily [6]. Farmers tend to keep the food products in the warehouses for a long period before sending them to the market, causing food wastage in large quantities since one spoilt batch usually spoils the entire batch. The farmers incur heavy losses which in turn leads to increased prices thus, adversely affecting the economy. This is one of the major reasons for the fluctuating prices of fruits and vegetables.
Earlier research has been done on the detection of different ripening stages of bananas using Computer Vision [9]. For example, a camera system has been proposed to observe the peel color on the surface of the fruit as an additional feature. The dual E-nose/camera system depicts the Fisher class separability measure. It displays a perfect classification of the four maturity stages of a banana: Unripe, half-ripe, fully ripe, and overripe [11]. In another method, the Raspberry Pi 3 Model B was used to collect and display the data from a sampling and sensor chamber. The sensor chamber consisted of 7 types of $\mathrm{MQ}$ sensors that were detecting the gas being released from tomato-based Filipino cuisine [2].

But these methods will be computationally expensive and memory exhaustive for storing the data and applying machine learning algorithms. Instead, we have approached the problem using temperature, humidity, and gas sensors that detect the emission of combustible gases from the food products (e.g., Banana and Tomato). Using this method, we obtained numeric values 
to build the dataset ourselves. The MQ2 gas sensor was linked to the Blink loT application using the ESP8266 module. The banana is placed in the sample chamber and the sensors capture data every 30 seconds. The values are automatically logged into a CSV file. We noticed an incremental graph of the gas concentration over a 5 days course. Based on the obtained data, we applied machine learning algorithms: Logistic regression, Decision Tree Classifier, Support Vector Classifier (SVC) \& K-Nearest Neighbours (KNN) classifier to predict if a banana is stale or not. We achieved an accuracy of $95.9 \%$ with the Support Vector Classifier. The same procedure is being applied to other fruits and vegetables too.

The system can be used in warehouses for food storage by manufacturers, the food processing industry, and supermarkets to monitor food quality in real-time and help the consumer gain complete transparency about the food they consume [1]. If people know exactly when their food is going to spoil, they can consume, donate, or convert it to compost. This way, food wastage reduces tremendously and the traditional methods of discarding food (which leads to increased Co2) can be avoided. The Agro-benefits can be seen where a farmer can predict and plan the distribution of his produce. As soon as one batch of the product is ready and ripe, the farmer will be notified, and he can quickly sell his product. The system will also notify him if one batch becomes overripe so that the other products can be saved. This will also minimize fluctuations in the global food economy. Overall, the project holds multiple benefits in addition to the above that can also be explored.

Fruits like bananas are usually stored together in a warehouse, a ripening wave is initiated due to the ethylene gas being emitted from the first banana [10]. This process expedites the ripening of other bananas as well, thus leading to the over-ripening of food products sooner than expected. Thus, constant monitoring is required to reduce food wastage.

\section{LITERATURE SURVEY}

The solution to cur pollution by monitoring air quality is presented in the form of a prototype that uses semiconductor gas sensors like MQ4 and MQ7. MQ7 can be used to detect carbon monoxide concentration in the range of 20 to $2000 \mathrm{ppm}$. The tin dioxide of the sensor reacts with carbon monoxide which results in the reduction of the sensor resistance. This reduction is an indication of an increase in the output voltage, which can now be measured. MQ4 sensor is used to detect natural gases like methane in the concentration range of 200 to 10,000 ppm. An LM35 sensor is also being used to obtain the output directly in the centigrade scale. Further, air quality was estimated based on Air Quality Index (AQI) guidelines set by the Central Pollution Board (CPCB). However, the prototype needs to be implemented on a larger scale to make an impact in reducing pollution. Additional ways to curb pollution must be suggested. In future systems, alerts using SMS or E-mail can be provided [3].

In this paper, the development of a small-size, lowcost portable e-nose has been discussed. Metal oxide semiconductors by Figaro have been used for the sensor array. PIC18F4520 is used in the Embedded E-nose and RS 232 is used to interface it to the PC for the acquisition, storage, and analysis of the data set consisting of odor signals from oranges and onions. The PIC18f4520 is used to control the sensor heaters and record the sensor response. It is interfaced to the sensor array consisting of 10 metal oxide semiconductor (MOS) sensors like TGS-813 and TGS-816 as well as temperature and humidity sensors like LM-35 and SYHS-220 respectively using programmable gain amplifiers (PGA). Four quads op-amp LM 324 IC is used as a unity gain amplifier as the signal conditioning unit to avoid loading. It was observed that the responses to food like onion and rotten onion were different. Thus, showing that this Embedded E-nose has shown promising results. However, pattern recognition algorithms still need to be developed and applied to identify the odor and freshness of food [4].

This project proposed a metal oxide semiconductorbased cost-effective E-nose that was able to differentiate between the different ripening stages of a banana. It was able to identify the difference between the aroma fingerprint of bananas and achieved an accuracy of $98.66 \%$ accuracy using Support Vector Machines (SVM). The experimental setup included four stages: (1) a sampling system, (2) a gas sensor array, (3) a data acquisition system, and (4) pattern recognition algorithms. 15 bananas varying in color, size, weight was placed inside the sampling chamber where the gas level (ethylene) and temperature were being controlled and monitored. The cycloid gas chamber consisted of 6 MOS sensors (MQ-3, MQ-5, MQ-9, MQ-131, MQ135, MQ-136), and using the data acquisition card, the gas sensor readings were stored in the computer and used for analysis to extract vital information. Principal component analysis (PCA), linear discriminant analysis (LDA), SIMCA, and SVM were the methods used for data analysis. SVM separates the classes using a hyperplane, which maximizes a quantity called margin and can be used for non-linear data as well. Thus, a low-cost MOSbased e-nose was designed to identify and differentiate between the different ripening stages of an e-nose. However, further work to develop additional sensors and an estimate about the right number of sensors need to be determined [7].

The review discusses the application of E-nose and E-tongue in determining properties that are related to food quality. Various sensors used in E-nose and tongue are compared based on the working principle, application, and limitation. Gas sensors like conducting polymers (CP), metal-oxide-semiconductor (MOS), quartz crystal microbalance (QCM), and surface acoustic wave (SAW) sensors can be used to develop an E-nose. These 
sensors then react with the target chemicals and cause irreversible changes in electrical properties like conductivity. MOS sensors are sensitive to hydrogen and unsaturated hydrocarbons and used metal oxide semiconductors like iron or zinc oxide. However, these sensors consume a high amount of energy as they have constraints related to working temperature conditions (between 150 to $400{ }^{\circ} \mathrm{C}$ ). CP sensors operate without external heating and hence consume less power than MOS sensors. However, these are sensitive to humidity and temperature and hence, it becomes necessary to maintain the optimum conditions at all times.

The properties of food samples can be recognized using machine learning classifiers and algorithms like PCA and SVM. In some cases, it has been combined with other classifiers to determine food quality. The decision tree used ID3 as a training algorithm. It builds the decision tree using the top-to-bottom and greedy approach. Hence, decision trees and RF can also be used to determine certain aspects related to food quality. Gas sensors are in general, extremely sensitive to temperature and humidity and sample preparation and sampling both depend on these factors. Even the sample size has to be considerably large to achieve a high level of accuracy. E-nose also consumes a high level of energy which thus, hampers their ability. Only time-invariant information could be detected as ANN and PCA were both developed for static features. Hence, an E-nose, smaller in size, unaffected by external environmental features, consumes less energy and still gives a high level of accuracy, needed to be developed. In addition to this, E-nose had to be made more user-friendly [13].

In this project, an e-nose (PEN2, Airsense Analytics, $\mathrm{GmBH}$, Germany) which consists of an auto-sampling apparatus, a sensor array, and a pattern recognizer software was used to detect the freshness of pork. The sensor array is made of ten MOS sensors lik3 W1C, W6S, and W3C. Factors like the mass of the pork samples, storage time, and headspace-generation time were used in the sampling procedure to determine the optimum experimental conditions. Linear Discriminant Analysis (LDA) and Back Propagation Neural Network (BPNN) were used to classify the pork samples based on storage times (ST) and showed an accuracy of 97.14\%. Multiple Linear Regression was used to predict the sensory scores. The correlation coefficients ( $R 2=0.9848)$ between the sensory scores and e-nose signal were high, thus proving that it can effectively assess pork freshness. The result showed that sensory scores of odor and viscosity reduced as storage time increased, especially after the third day. Thus, the algorithms applied were able to classify the pork samples during the storage period, thus predicting the storage time and sensory scores. They were well discriminated by LDA, classified correctly by BPNN and the quality index was provided by MLR. However, the PEN2 e-nose is comparatively expensive and not easily available. Also, additional Machine Learning algorithms need to be developed to increase accuracy and range [5].

In this paper, Electronic noses are being developed for the automated identification of volatile chemicals. They have described and developed a basic prototype of an Electronic Nose and discussed its' applications in the field of medicine, food, and the environment. Electronic Nose consists of a sensor array and Artificial Neural Networks (ANN). Each chemical vapor makes a particular signature/pattern when detected by a sensor. Introducing different chemical vapors will create a database of those signatures. ANN is then used to analyze the complex database and identify the chemical vapor. A prototype consisting of nine tin-oxide vapor sensors along with temperature and humidity sensors was built. The ANN used for this prototype was a multilayer feed-forward network trained with the backpropagation algorithm and the fuzzy ARTmap algorithm. This prototype was tested on five chemicals: acetone, ammonia, isopropanol, lighter fluid, and vinegar. The two networks were trained using randomly selected training patterns and an accuracy ranging from $89.7 \%$ to $98.2 \%$ was achieved. In the medical field, an electronic nose detects possible problems by analyzing odors from the human body. Using this can also reduce the amount of analytical chemistry used in food production and its applications are in assessing food quality, checking rancidity of mayonnaise, and grading of whiskey. However, it still needs to compare the prototype with the conventional systems as well as develop a field system. Also, additional neural networks should be applied to ensure the highest level of accuracy [14].

The ripeness of postharvest kiwifruit was predicted using an E-nose with 10 MOS sensors. Three different feature extraction methods like max/min values, difference values, and 70th values were used to discriminate the fruit at different stages. In addition to this, Partial least squares regression (PLSR), SVM, and random forest (RF) were used to predict overall ripeness, soluble solids content (SSC), and firmness. 160 kiwifruits were subdivided into groups of 20 each and stored in an incubator $\left(20{ }^{\circ} \mathrm{C}\right.$ and $70 \%$ relative humidity). PEN3 E-nose system was used to detect the gas from the samples. The E-nose was preheated to reach working temperature and sensor 2 (W5S) gave the most significant response. Further, SSC and firmness were used to determine the sweetness and hardness of kiwifruit. The three features were used along with LDA to classify the different ripening stages. RF algorithm showed the best performance with overall ripeness (training: $\mathrm{R} 2=0.9928$; testing: $\mathrm{R} 2=0.9928$ ), SSC (training: $\mathrm{R} 2=$ 0.9749 ; testing: $R 2=0.9143$ ) and firmness (training: $R 2$ $=0.9814$; testing: $R 2=0.9290)$. Thus, this study successfully predicted the ripeness of postharvest kiwifruit using a MOS E-nose using different feature extraction and pattern recognition methods [8].

The study aims to determine the effectiveness of a fast gas chromatography (GC) e-nose which is used to 
determine the storage time and internal quality changes during the storage of hen eggs. 3 experiments were conducted: egg volatile detection, internal quality measurement, and sensory evaluation. Yolk and albumen color, spread ratio, freshness, and overall acceptability were the evaluated sensory characters. The fast GC e-nose consists of a sampling system (HS100 autosampler), a detector system connected to 2 flame ionization detectors (FID), and a data acquisition and processing system. Observations showed that haugh unit values decreased as the storage time increased due to a decrease in albumen height. Principal component analysis and Discriminant Factor Analysis were further used to process the data. These confirmed the difference in volatile profiles of egg samples and accounted for a total variance of $95.7 \%$ and $93.71 \%$, respectively. Thus, it was concluded that the fast GC e-nose can be used as a reliable instrument for determining the prediction and quality assessment of egg freshness, especially during the supply-chain phase [12].

\section{BLOCK DIAGRAM}

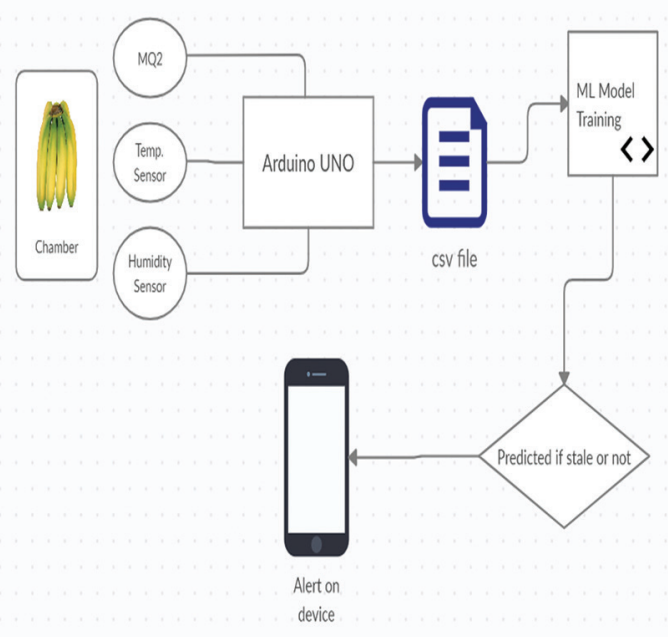

Fig. 1. Block Diagram

\section{IMPLEMENTATION}

Being a novel idea, there were not any datasets readily available that were structured or could be structured as per our requirement. So, we decided that we would be building a dataset on our own using a banana and a few sensors. The stages of implementation are explained below.

\subsection{PROOF OF CONCEPT}

The traditional E-noses available in the market are either too expensive, inefficient, and difficult to use. Hence, the first stage involved developing a cost-effective version of the e-nose. After thorough research, the MQ2 sensor, a metal oxide semiconductor was used to test the food products for gas level concentrations (mainly ethylene).
The MQ2 sensor is conventionally used for smoke detection. However, we observed and identified that it is highly sensitive to combustible gases. Concentrations of the gas are measured using a voltage divider network present in the sensor. This sensor works on $5 \mathrm{~V}$ DC voltage. It can detect gases in the concentration of range 200 to 1000 ppm. This sensor contains Ceramic coated with Tin dioxide, enclosed in a stainless-steel mesh. The sensing element has six legs attached to it, two of which are responsible for heating the sensing element and four are used for output. Oxygen gets absorbed on the surface of sensing material when it is heated in air at a high temperature. The donor electrons present in tin oxide are attracted towards this oxygen, thus preventing the current flow. The ethylene gas from the banana reacts with these oxygen atoms, thereby decreasing the surface density of absorbed oxygen. Current can now flow through the sensor which generates the analog voltage values. This voltage value is an indicator of the concentration of the ethylene gas. A higher concentration of the gas results in a higher analog voltage and vice-versa.

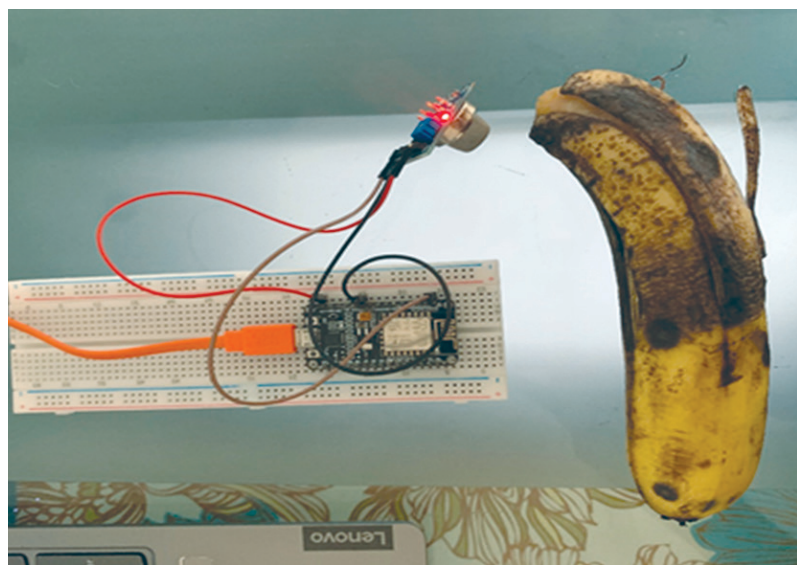

Fig. 2. Proof of Concept Stage

ESP8266 was used to interface the MQ2 gas sensor array with the Blynk loT application to observe the data in real-time. A banana was put under test and the gas concentrations released by the banana as it continued to ripen were observed. With an increase in time, the gas concentrations increased significantly thus proving that the MQ2 sensor could be used to develop a costeffective version of an E-nose.

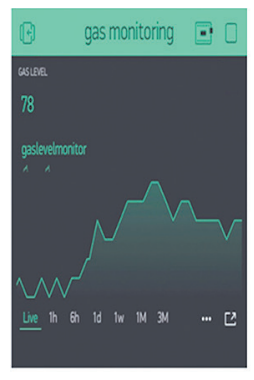

DAY 1:GASLEVEL AT 78

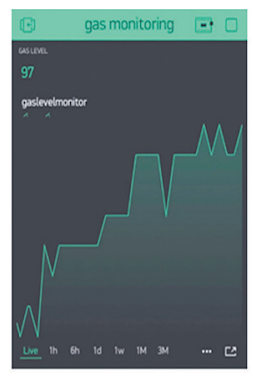

DAY 2: GASLEVELAT 97

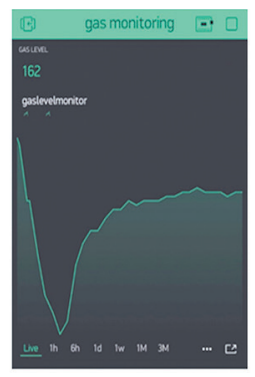

DAY 3:GASLEVEL AT 162
Fig. 3. Graphs for day 1 to 3 


\subsection{SAMPLING CHAMBER}

The gas chamber is an enclosed, air-tight, and controlled environment where the food products have been tested. The chamber must be cleaned at regular intervals with a clean cloth. The controlled environment ensured accurate data collection and reduced fluctuations. It is essential that the chamber remains airtight as outside parameters can severely affect the operating conditions and working of the sensor which is highly sensitive. The gases accumulated in the chamber must be released after every testing interval. The temperature and humidity data are collected using DHT11 sensor that is important to get higher accuracy in the machine learning stage by providing additional attributes.

The gases being released from the product get accumulated in the chamber over time, aiding in the ripening of the food products. Thus, as observed in the'proof of concept' stage, the gas concentrations being released from the banana keeps on increasing exponentially. Based on this observation, concerned individuals must release batches of their products to ensure that the rest of the product remains fresh.

\subsection{DATA COLLECTION}

The MQ2 gas sensor is interfaced with an Arduino Uno Board as it is highly compatible and easy to code using C Language on Arduino IDE software. The Arduino Uno reads the analog output voltage at one of its pins, connected directly with the gas sensor. The temperature and humidity sensor, DHT11 was also connected and calibrated to the Arduino analog pins using a similar procedure. This ensured constant monitoring of the room conditions and took into consideration both temperature and humidity, in real-time. 3000 samples over a period of 2 days were taken at a rate of 30 seconds per sample.

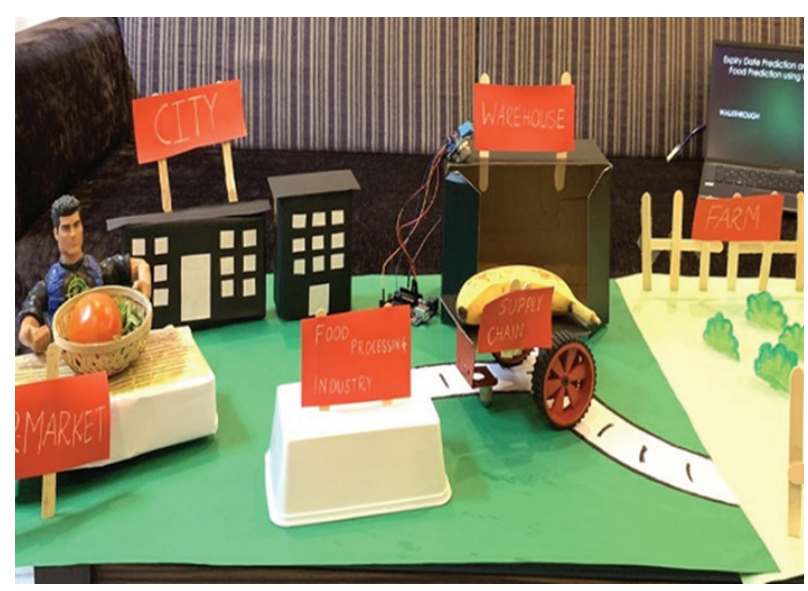

Fig. 4. 3D Model Implementation

\subsection{DATA PREPARATION}

An unripe banana was taken and placed inside the chamber. The MQ2 gas sensor, also placed inside the compact chamber, was activated by supplying a $5 \mathrm{~V}$ DC supply through an Arduino module. Similarly, the temperature and humidity sensors were connected and calibrated to the Arduino board. The gas sensor was heated for a few minutes before we started to take the readings. The data from the sensors were captured every 30 seconds. Using the comma as the delimiter, the values were printed onto the serial monitor of an application named Teraterm, which directly logged the data into a CSV file. After repeating this procedure multiple times at different hours of the day, sufficient data had been created in different CSV files. All the CSV files were then merged with their matching columns to create one huge dataset that could further be used for training our machine learning model.

Metal Oxide Semiconductor (MOS) uses a simple voltage divider network. When the gas comes in contact with the sensing element made of Aluminum Oxide (Al2O3) and coated with Tin Dioxide (SnO2), the resistance of the voltage divider network changes in proportion to the gas concentrations. In a clean environment, the electrons in the tin dioxide layer are attracted towards the oxygen present on the surface of the sensing material. However, oxygen present on the surface decreases as they react with certain combustible gas particles thus allowing the donor electrons to become free and allowing a flow of current through the sensor.

MOS sensors operate in the temperature range of 200 to $400^{\circ} \mathrm{C}$. These sensors are highly reliable, stable, sensitive, and have a long lifetime. These can be operated in the wide temperature range of $-40^{\circ} \mathrm{C}$ to $+70^{\circ} \mathrm{C}$ and humidity range of 0 to $100 \% \mathrm{RH}$. They have a short response and recovery time and do not require heavy maintenance.

\subsection{PROPOSED SYSTEM}

We have used python for data handling, data preprocessing, and training our model. Google Collaboratory with a GPU-based runtime environment was utilized to write and execute our python code. To work with the imported CSV file, we used common python libraries. First, we converted the CSV file into a data frame using the Pandas library. One of the datasets which contain 3000 data points is displayed in Table 1 given below, where we have printed only the first 10 values (head) of the dataset for initial analysis. We can see the data frame consists of 4 features: gas concentration (in ppm), temperature value, and humidity values are the input features whereas the last column (overripe/ripeness) is the target value that needs to be predicted by our model. Additionally, we used the seaborn library for plotting and visualizing the data we had acquired which helped us gain some useful insights. The graph plot for gas concentration value can be seen in Fig. 6. 
Table 1. Data Frame (first 10 data points)

\begin{tabular}{ccccc}
\multicolumn{5}{c}{ Different Features/Parameters present in Dataset } \\
Index & Gas (ppm) & Temperature & Humidity & Ripeness \\
\hline 0 & 0.083333 & 0.456405 & 0.791574 & 0 \\
\hline 1 & 0.083333 & 0.627228 & 0.595237 & 0 \\
\hline 2 & 0.083333 & 0.598781 & 0.599076 & 0 \\
\hline 3 & 0.083333 & 0.422635 & 0.702421 & 0 \\
\hline 4 & 0.083333 & 0.618524 & 0.829969 & 0 \\
\hline 5 & 0.083333 & 0.247694 & 0.752487 & 0 \\
\hline 6 & 0.083333 & 0.568728 & 0.688531 & 0 \\
\hline 7 & 0.083333 & 0.470279 & 0.785262 & 0 \\
\hline 8 & 0.083333 & 0.520554 & 0.839753 & 0 \\
\hline 9 & 0.083333 & 0.616535 & 0.689238 & 0 \\
\hline
\end{tabular}

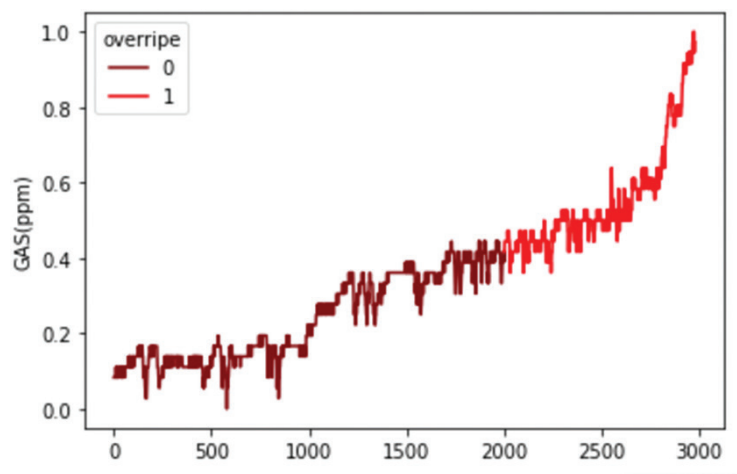

Fig. 5. Gas Concentration Plot

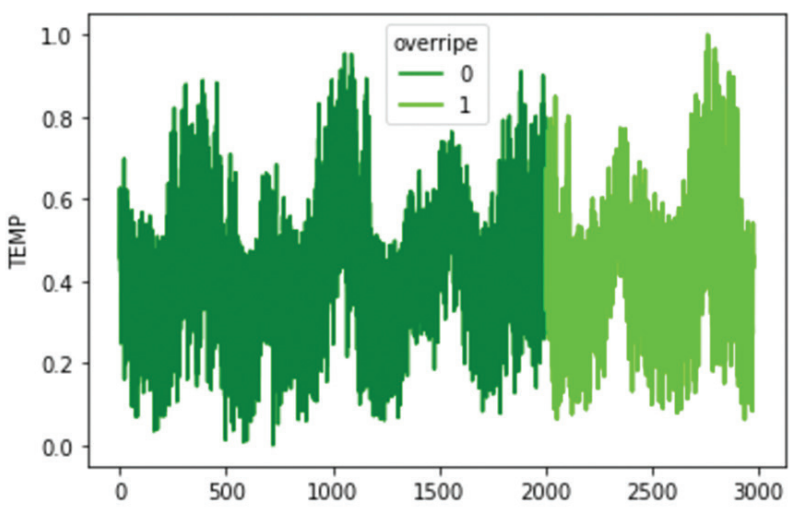

Fig. 6. Temperature Variations

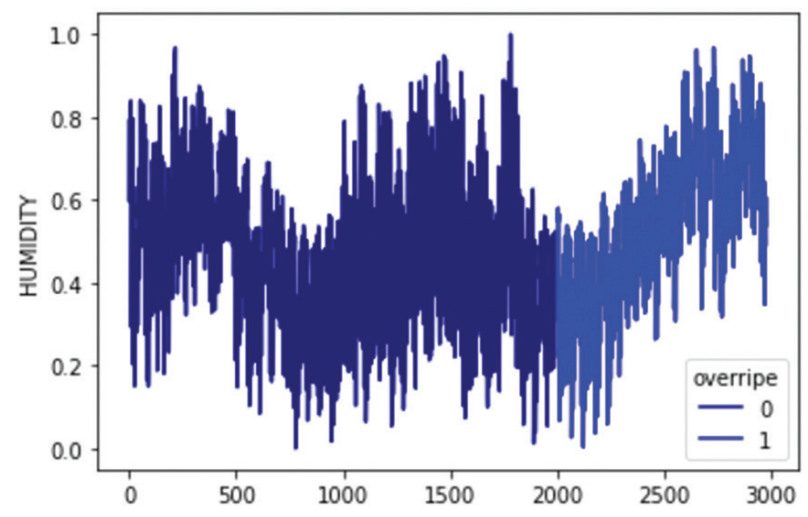

Fig. 7. Humidity Variations

\subsection{STATIONARITY}

The gas concentration value is non-stationary as it keeps on increasing with time. Whereas the humidity and temperature values are stationary and periodic as the values kept on oscillating between a particular range of values. We can also observe that the gas concentration value starts to increase exponentially after a certain threshold as there is an increase in the emission of ethylene gas as the fruit starts to ripen.

\subsection{DATA CLEANING (PREPROCESSING)}

By observing the data plot, some outliers can be seen present in the line-plot because the gas sensor is sensitive to temperature and humidity changes. The values which created abrupt changes and disturbed the uniform linear growth of the gas concentration were termed as outliers and removed from the dataset. Secondly, when the fruit is unripe there is no significant change in the concentration leading to a flat graph. Hence, we decided to discard the starting values till they did not begin to show significant changes.

\subsection{MODEL TRAINING}

For shuffling and splitting the dataset, creating, and training our machine learning model, and finally calculating the accuracy of the different models, we used the Scikit learn module which has multiple libraries available to carry out these functions. The dataset was shuffled randomly so that range of values is properly distributed before splitting it to the training set $(75 \%)$ and test set $(25 \%)$. As the numerical features like gas concentration, humidity and temperature have a different range of values, to avoid bias in the data, we used StandardScaler (a Sklearn library) to scale the data to a given range. It can be inferred from the given table below, Logistic regression, Decision Tree Classifier, Support Vector Classifier (SVC) \& K-Nearest Neighbours (KNN) classifier were the different models trained and tested on our data. We achieved the highest accuracy of $97.05 \%$ on the Support Vector Classifier model.

\subsection{DATA COLLECTION}

The traditional E-noses available in the market are either too expensive, inefficient, and difficult to use. Hence, the first stage involved developing a cost-effective version of the e-nose. After thorough research, the MQ2 sensor, a metal oxide semiconductor was used to test the food products for gas level concentrations (mainly ethylene).

\section{RESULTS}

\subsection{RESULT TABLE}

Following is the accuracy and ROC_AUC score obtained for different machine learning models. Accuracy Score column measures how many observations, both ripe and unripe, were correctly classified. 
ROC AUC Score tells us how good our model is by calculating the Area Under the ROC Curve (Refer Fig. 4). More top-left the ROC curve is, the higher the area and hence we will get a higher ROC AUC score.

Table 2. Accuracy and ROC AUC Score of different models

\begin{tabular}{|ccc|}
\hline Machine Learning Model & Accuracy Score & ROC AUC Score \\
\hline Decision Tree Classifier & $96.51 \%$ & 0.992 \\
\hline Gaussian Naïve Bayes & $91.3 \%$ & 0.987 \\
\hline KNN Classifier & $95.97 \%$ & 0.989 \\
\hline Logistic Regression & $96.78 \%$ & 0.995 \\
\hline Support Vector Classifier & $97.05 \%$ & 0.996 \\
\hline
\end{tabular}

\subsection{LEARNING CURVES}

The learning curves are computed to choose the perfect ratio to split the dataset into training and testing sets, to minimize the error, improve the accuracy of the model and allow to detect of high variance or high bias is present in the data. After careful evaluation of the learning curves, the dataset was split with $75 \%$ of the data as a training set and $25 \%$ as the test set.

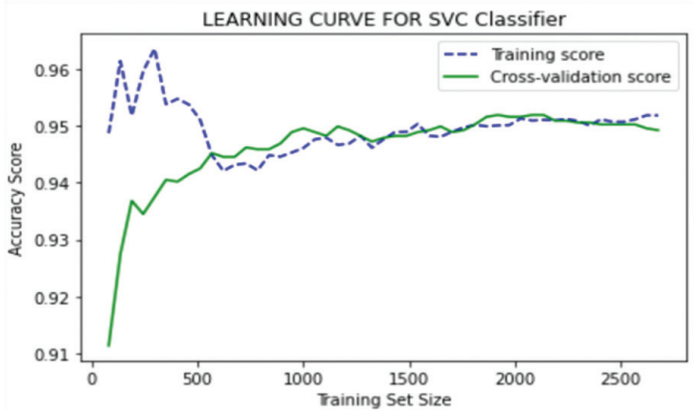

Fig. 8. Gas Concentration Plot

\subsection{ROC CURVES}

The Receiver Operating Characteristic curve (ROC) curve is a useful tool for predicting the probability of a binary outcome, which plots the false positive rate ( $x$ axis) versus the true positive rate (y-axis) for different candidate threshold values between 0.0 and 1.0. ROC curves are to be used when the observations are balanced between each class.

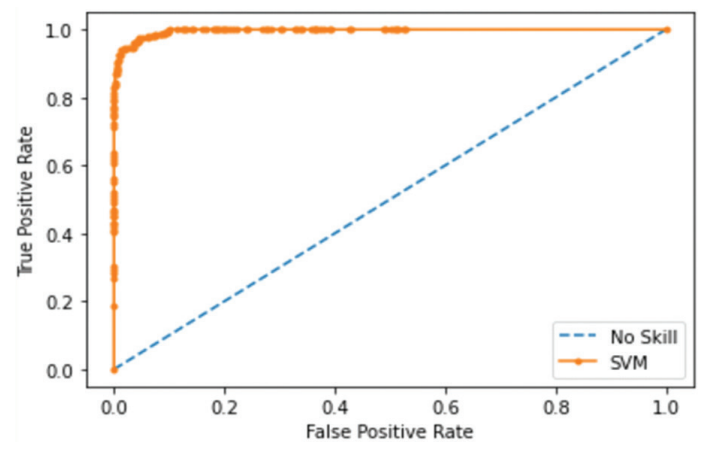

Fig. 9. The curve for SVM Classifier

\subsection{CONFUSION MATRIX}

The confusion matrix represents the number of correct and incorrect predictions made by the classifier, thereby evaluating the performance of the classification model. The matrix is displayed in the form of a heatmap.

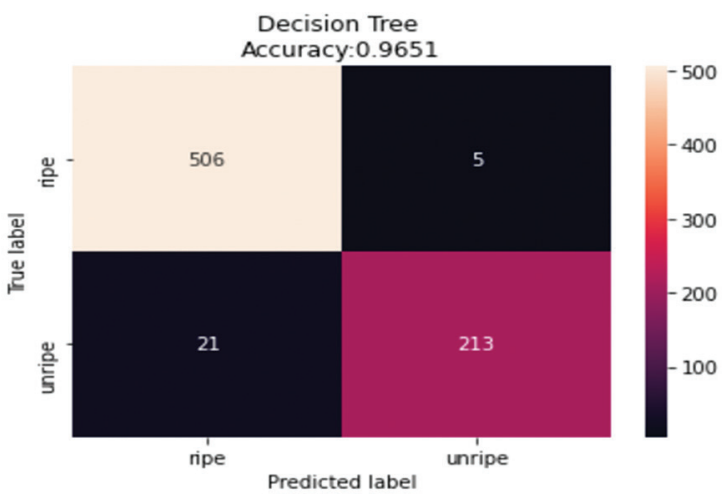

Fig. 10. Confusion Matrix

\section{CONCLUSION}

Technologies like the Internet of Things (IoT) and Machine Learning have been used in this project to accurately determine the expiry date of food products to reduce food wastage. To achieve this, a cost-effective version of a traditional e-nose was developed and used for the detection of staleness of food products. The gas level (ethylene) was mapped and recorded for different ripening stages and a data set was recorded successfully using a banana. After the data collection, data cleaning (prep-processing steps) was implemented. Further, machine learning algorithms like Logistic regression: 96.78\%, Decision Tree Classifier: 96.51\%, Support Vector Classifier (SVC): $97.05 \%$ \& K-Nearest Neighbours (KNN) classifier: $95.97 \%$ were used to process the data and gain valuable insights regarding the ripening stage and expiry date. These results proved that this low-cost e-nose, supported by efficient machine learning algorithms can be used to determine the expiry date and reduce food wastage.

\section{FUTURE SCOPE}

The project model used and discussed in this paper can be extended to work with any food product that emits incremental amounts of ethylene gas or other volatile gases during its ripening stages. The current sensor-based system can be enhanced by using Raspberry $\mathrm{Pi}$ and camera-based model. The camera can be used to detect the color and texture of the food item while the $\mathrm{R}$-Pi will provide additional processing capability. The machine learning models mentioned above are only being used as a binary classification algorithm to predict if the food item is getting stale or not. It can be further enhanced by utilizing a time-series forecasting model which can show accurate expiration dates of products 
beforehand. The prototype can be made compact and easy to use by individuals in different industries like food processing, supply chain, and farming. It can be used to raise awareness among people about the accurate expiry date of their food products. We also want to provide suggestions on the accurate way to utilize/dispose of their food products, for example, if it has reached an over-ripened state, the suggestions regarding composting will be provided to the user via a mobile notification, thus ensuring that the food is not wasted, but utilized optimally.

\section{REFERENCES}

\section{Conference papers:}

[1] H. Vaviya, A. Yadav, V. Vishwakarma, N. Shah, "Identification of artificially ripened fruits using machine learning", Proceedings of the $2^{\text {nd }}$ International Conference on Advances in Science \& Technology, Mumbai, India, 8-9 April 2019.

[2] M. V.C Caya. et al., "Determining spoilage level against time and temperature of tomato-based Filipino cuisines using electronic nose", Proceedings of the IEEE $9^{\text {th }}$ International Conference on Humanoid, Nanotechnology, Information Technology, Communication and Control, Environment and Management, Manila, Philippines, 1-3 December 2017.

[3] S. Karamchandani, G. Aaklin G. Deven, "Pervasive monitoring of carbon monoxide and methane using air quality prediction", Proceedings of the 3rd International Conference on Computing for Sustainable Global Development, New Delhi, India, 16-18 March 2016.

[4] A. Kumbhar, D. C. Gharpure, B. A. Botre, S. S. Sadistap, "Embedded e-nose for food inspection", Proceedings of the 1st International Symposium on Physics and Technology of Sensors, Pune, India, 7-10 March 2012.

[5] X. Hong, J. Wang. "Discrimination and Prediction of Pork Freshness by E-nose", International Conference on Computer and Computing Technologies in Agriculture, Beijing, China, 29-31 October 2011.

\section{Journal Papers:}

[6] M. Alongi, S. Sillani, L. Manzocco, "Effect of expiry date communication on acceptability and waste of fresh-cut lettuce during storage at different temperatures", Food Research International, Vol. 116, 2019, pp. 1121-1125.

[7] A. Sanaeifar, S. S. Mohtasebi, M. Ghasemi-Vanamkhasti, H. Ahmadi, J. Lozano, "Development and application of a new low-cost electronic nose for the ripeness monitoring of banana using computational techniques (PCA, LDA, SIMCA, and SVM)", Czech Journal of Food Sciences, Vol. 32, No. 6, 2014, 538-548

[8] D. Du, Dongdong, J. Wang, B. Wang, L. Zhu, Z. Hong, "Ripeness prediction of postharvest kiwifruit using a MOS E-Nose combined with chemometrics", Sensors, Vol. 19, No. 2, 2019, p. 419.

[9] S. Gunasekaran, "Computer vision technology for food quality assurance", Trends in Food Science \& Technology, Vol. 7, No. 8, 1996, pp. 245-256.

[10] S. P. Burg,.E. A. Burg, "Ethylene action and the ripening of fruits: Ethylene influences the growth and development of plants and is the hormone which initiates fruit ripening", Science, Vol. 148, No. 3674, 1965, pp. 1190-1196.

[11] L.-Y. Chen, C.-C. Wu, T.-I. Chou, S.-W. Chiu, K.-T. Tang, "Development of a Dual MOS electronic nose/camera system for improving fruit ripeness classification", Sensors, Vol. 18, No. 10, 2018, pp. 3256.

[12] S. M. Yimenu, J. Y. Kim, B. S. Kim, "Prediction of egg freshness during storage using an electronic nose", Poultry Science, Vol. 96, No.10, 2017, pp. 3733-3746.

[13] J. Tan, J. Xu, "Applications of electronic nose (enose) and electronic tongue (e-tongue) in food quality-related properties determination: A review", Artificial Intelligence in Agriculture, Vol. 4, 2020, pp. 104-115.

[14] P. E. Keller, "Electronic noses and their applications", Proceedings of the IEEE Technical Applications Conference and Workshops, Portland, OR, USA, 10-12 October 1995. 